\title{
Biomechanical Evaluation of Anterolateral Screw Fixation for Oblique Lumbar Interbody Fusion Surgery - A Finite Element Analysis
}

gufang Fang

Orthopedic Institute

SG Chen

Orthopedic Institute

wda zhuang

Orthopedic Institute

WH Huang

Orthopedic Institute

Hongxun Sang ( $\nabla 189962700 @ q q . c o m)$

orthopedics

Research article

Keywords: OLIF, anterolateral screw fixation, Pedicle screw fixation, Spinal fusion, Finite element analysis

Posted Date: October 9th, 2020

DOI: https://doi.org/10.21203/rs.3.rs-71475/v1

License: (c) (1) This work is licensed under a Creative Commons Attribution 4.0 International License.

Read Full License 


\section{Abstract}

Background: The most common complication of oblique lumbar interbody fusion (OLIF) is cage subsidence. OLIF combined with internal fixation could help decrease the cage subsidence and increase the fusion rate. The aim of this study was to evaluate the biomechanical feasibility and safety in the patients undergoing OLIF surgery with anterolateral screw fixation (ASF).

Methods. Based on our previous validated model , L4-L5 functional surgical models corresponding to the ASF and Bilateral pedicle screw fixation(BPSF) methods were created. A $500 \mathrm{~N}$ compression force was applied to the superior surface of the model to represent the upper body weight, and a $7.5 \mathrm{Nm}$ moment was applied to simulate the six movement directions of the lumbar spinal model: flexion/extension, right/left lateral bending and right/left axial rotation. Finite element (FE) models were developed to compare the biomechanics of the ASF and BPSF groups.

Results. Compared to the range of motion (ROM) of the intact lumbar model, that of the ASF model was decreased by $82.0 \%$ in flexion, $60.0 \%$ in extension, and the BPSF model was decreased by $86.7 \%$ in flexion, $77.3 \%$ in extension. Compared to the BPSF model, the maximum stresses of the L4 inferior endplate (IEP) and L5 superior endplate (SEP) were greatly increased in the ASF model; The contact surface between vertebrae and screw (CSVS) in the ASF model produced nearly $100 \%$ more stresses than the BPSF model in all moment .

Conclusions. OLIF surgery with ASF could not reduce the maximum stresses on the endplate and CSVS, which may be a potential risk factor for cage subsidence and screw loosening.

\section{Background}

Oblique lumbar interbody fusion(OLIF) was introduced in 2012 by Silvestre [1]. The stand-alone (SA) procedure is associated with a low risk of post treatment trauma or bleeding and offers good stability and quick recovery. However, complications associated with this technique have been frequently reported [2-5]. The most common complication was endplate fracture/subsidence (18.7\%) [6]. Malham [7] found that for patients with osteoporosis, the combination of internal fixation could help decrease the cage subsidence and increase the fusion rate. OLIF combined with bilateral pedicle screw fixation (BPSF) significantly increased the operative time, medical expenditure and risks associated with pedicle screw placement [8]. Anterolateral screw-rod fixation (ASF) procedure enabled to reduce the operative time, blood loss, radiological exposure, and soft tissue disruption, because instrumentation could be applied through the same surgical corridor without extra incision and exposure[9] .But the stability and safety of OLIF surgery with a ASF procedure have never been explored. Few literatures reported the biomechanical evaluation about the OLIF with ASF.

Recently, FEA of lumbar biomechanics had become an important test for the evaluation of surgical feasibility and the design of instruments. [10]. The purpose of this study was to evaluate stability and safety of OLIF surgery with ASF by Finite element analysis (FEA). 


\section{Methods}

\subsection{Development of the OLIF FE model}

Base on our previous validated model[11], an OLIF cage (10 mm in height, $55 \mathrm{~mm}$ in length, $18 \mathrm{~mm}$ in width ,5-degree sagittal angle) was assembled based on the L4-L5 functional spinal unit (FSU) model (Fig. 2) to simulate the SA model. Two screws $(6.5 \mathrm{~mm}$ in diameter and $45 \mathrm{~mm}$ in length) and one rods (5.5 $\mathrm{mm}$ in diameter and $45 \mathrm{~mm}$ in length) were assembled on left lateral sides of the SA model to simulate the ASF model, and four pedicle screws and two rods were assembled on both sides of the posterior of SA model to simulate the BPSF model (Fig. 1). The properties were also adopted from the literature (Table 1) [10]. The bottom of the L5 vertebral body was fully constrained(Fig. 1).

Table 1

Assigned Material Properties for the Finite Element Models

\begin{tabular}{|lllll|}
\hline Tissues & Modulus (MPa) & Poisson's ratio & Element type & Thickness \\
\hline Cortical bone & 12000 & 0.3 & Shell & $1 \mathrm{~mm}$ \\
\hline Cancellous bone & 100 & 0.2 & Solid & $/$ \\
\hline Bony endplate & 12000 & 0.3 & Shell & $0.8 \mathrm{~mm}$ \\
\hline Facet & 35 & 0.4 & Shell & $0.2 \mathrm{~mm}$ \\
\hline Annular ground substance & $\mathrm{c} 1=0.18, \mathrm{c} 2=0.045$ & $/$ & Solid & $/$ \\
\hline Nucleus pulposus & $\mathrm{c} 1=0.12, \mathrm{c} 2=0.03$ & $/$ & Solid & $/$ \\
\hline Annular collagen fiber & 450 & 0.3 & Surface & $/$ \\
\hline PEEK (polyetheretherketone) & 3700 & 0.3 & Solid & $/$ \\
\hline Titanium (Ti-6Al-4V) & 110000 & 0.3 & Solid & $/$ \\
\hline
\end{tabular}

\subsection{Finite element analysis}

The load process consisted of two steps. In the first step, a $500 \mathrm{~N}$ compression force was applied to the superior surface of the model to represent the upper body weight. In the second step, a moment of $7.5 \mathrm{~N} \mathrm{~m}$ was applied to the surface of the model to test the six movement directions of the lumbar spinal model: flexion/extension, right/left lateral bending and right/left axial rotation. The biomechanics of the SA,ASF and BPSF groups were compared to evaluate the risk of cage subsidence.

\section{Results}

\subsection{ROM and displacement in the OLIF model}


Compared to ROM of the intact lumbar model, that of the SA model was decreased by $79.6 \%$ in flexion, $54.5 \%$ in extension, $57.2 \%$ in lateral bending, and $50.0 \%$ in axial rotation, and the ASF model was decreased by $82.0 \%$ in flexion, $60.0 \%$ in extension, $74.0 \%$ in left lateral bending and $68.8 \%$ in right lateral bending, $72.2 \%$ in left axial rotation and $71.4 \%$ in right axial rotation, and the BPSF model was decreased by $86.7 \%$ in flexion, $77.3 \%$ in extension, $76.2 \%$ in lateral bending, and $75.0 \%$ in axial rotation. (Fig. 2 ). These results showed that the OLIF procedure with BPSF could considerably reduce the ROM of the fusion segment. However, OLIF with the SA procedure could not effectively reduce the extension, lateral bending and axial rotation motion, and OLIF with the ASF procedure could not effectively reduce the extension motion (no more than $60 \%$ ).

\subsection{Stress in the models}

Compared to the SA model, the maximum stresses of L4 IEP and L5 SEP were significantly decreased in the BPSF model; the L4 IEP max stress was decreased to $11.25 \mathrm{MPa}$ in extension, and the L5 SEP max stress was increased to $28.89 \mathrm{MPa}$ in flexion (Fig. 3, Fig. 4, Fig. 5 and Fig. 6). Compared to the SA model ,the maximum stresses of L4 IEP and L5 SEP were slightly decreased in the ASF model,L4 IEP max stress was $41.72 \mathrm{MPa}$ in extension and L5 SEP max stress was $40.51 \mathrm{MPa}$ in flexion (Fig. 4, Fig. 6). The L4 IEP of the SA model produced $339 \%$ greater stress than the BPSF model in the extension moment, and the L5 SEP of the SA model produced $64 \%$ greater stress than the BPSF model in the flexion moment. The L4 IEP of the ASF model produced $270.84 \%$ greater stress than the BPSF model in the extension moment, and the L5 SEP of the ASF model produced $40.22 \%$ greater stress than the BPSF model in the flexion moment. These results indicated that OLIF with SA and ASF was associated with a higher risk of endplate fracture/subsidence in the flexion and extension motions than OLIF with BPSF. OLIF with BPSF could greatly decrease the von Mises stress of the endplate, which may reduce the risk of endplate fracture.

The maximum von Mises stresses of the contact surface between vertebrae and screw (CSVS) were significantly increased in the ASF model in all moments compared to the BPSF model. The CSVS in the ASF model produced nearly $100 \%$ more stresses than the BPSF model in all moment (Fig. 7 and Fig. 8).The maximum von Mises stresses of CSVS were 33.68 MPa in ASF model and 16.45 MPa in BPSF model in flexion moment.

The maximum von Mises stresses of the screw increased slightly in the BPSF model in the flexion, bending and rotation moments compared to the ASF model. In the extension moment, The maximum stresses of the screw in the BPSF mode decrease 10.20\% compared to the ASF model (Fig. 9 and Fig. 10).The max stresses of screw were 141.7 MPa in flexion moment in BPSF model and $116.45 \mathrm{MPa}$ in ASF model(Fig. 10).

The maximum von Mises stresses of the cage increased slightly in the ASF model in the flexion, extension, right bending and rotation moments compared to the SA model and decreased $58.3 \%$ in the left bending moment. The max stresses of cage decreased largely in the BPSF model in all moments, especially in the extension moment, the max stresses decrease $83.9 \%$ compared to the SA model. In the 
extension moment, the max stresses of cage were 68.64 MPa in extension moment in SA model ,47.88 MPa in ASF model and 11.08 MPa in BPSF model (Fig. 11 and Fig. 12).

\section{Discussion}

OLIF surgery has become popular recent years. Nevertheless, complication rates range from $3.7-66.7 \%$ $[2-5,12-13]$. The cage subsidence incidence in the stand-alone group was higher than that in the OLIF combined with posterior pedicle screw fixation group [7]. OLIF surgery with bilateral pedicle screw fixation (BPSF) may be an alternative solution to reduce the complication [7]. Liu JP reviewed 15 patients underwent OLIF surgery with ASF and only 1 case of endplate damage (6.7\%) was founded and interbody fusion rate was 95\%[8]. Compare to BPSF procedure, ASF procedure could reduce the total operative time, blood loss, radiological exposure, and soft tissue disruption. It seemed OLIF with ASF was a promising procedure. But Biomechanical evaluation about the OLIF with ASF had never been reported.

Based on the our previous validated lumbar model[11], OLIF models including SA, ASF and BPSF procedures at the level of the FSU (L4-L5) have been developed. The simulation showed that BPSF could extensively reduce the ROM of the lumbar segment. However, SA OLIF procedure could not effectively reduce the extension, lateral bending and axial rotation motion of the lumbar segment (less than 60\%). ASF OLIF procedure could not effectively reduce the extension of lumbar segment (no more than $60 \%$ ).

Under $500 \mathrm{~N}$ of compression force and $7.5 \mathrm{~N} \mathrm{~m}$ moment loading, in the SA model, the maximum stress of the L4 IEP was 47.6 MPa in extension movement, the maximum stress of the L5 SEP was 46.2 MPa in flexion movement, and in the ASF model, in L4 IEP max stress was 41.7 MPa in extension and L5 SEP max stress was 40.5 MPa in flexion. Compared to OLIF with SA, the maximum stresses of L4 IEP and L5 SEP of OLIF with ASF were slightly decreased which suggested OLIF ASF could not reduce the cage subsidence effectively. The L4 IEP of the BPSF model exhibited $77.2 \%$ less stress than the SA model in extension moment, and the L5 SEP of the BPSF model showed $39.0 \%$ less stress than the SA model in the flexion moment; therefore, BPSF could efficiently reduce the maximum stress on the endplate. This indicated that OLIF with BPSF was safer than OLIF with SA and ASF regarding cage subsidence. The maximum von Mises stresses of CSVS were 33.68 MPa in ASF model and 16.45 MPa in BPSF model in flexion moment. The CSVS in the ASF model produced nearly $100 \%$ more stress than the BPSF model in all moment which meant CSVS of ASF procedure was easier to be broken than BPSF procedure in the patients with poor bone quality and had high risk of screw loosening. In clinical practice, Xie [14] reviewed 65 patients underwent OLIF surgery with ASF and 5 cases of cage subsidence (7.7\%) were founded. He concluded cage subsidence was the most common complication in the OLIF with ASF.

Overall, the FEA revealed that the SA and ASF procedure could not provide sufficient rigidity in OLIF surgery. Compared to OLIF with SA, OLIF with ASF could not reduce the max stresses of endplate and could not reduce the cage subsidence effectively.

In conclusion, from the FEA study, OLIF with ASF could not reduce the cage subsidence effectively. BPSF is important for OLIF surgery, although it is time-consuming and may result in other complications such 
as fixation failure, infection, and neurological deficits [15], compared to the greater risk of cage subsidence, the additional risks of BPSF are lower and less destructive.

From this study, for patients with excellent bone quality, OLIF with ASF procedure may be a good solution.

OLIF with ASF procedure is not suitable for patients with poor bone quality, such as osteoporosis, and have high risk of cage subsidence and screw loosening. OLIF with BPSF is the best choice for them. OLIF with BPSF is an ideal solution for patients with cage subsidence after undergoing OLIF with ASF (Fig. 13). The FEA does not provide actual clinical outcome evidence. Further clinical studies should be done in the future to verify the FEA results. Due to multiple risk factors for endplate fracture may exist, including endplate damage, obesity, high iliac crest, and poor stability of lesion segments [7], the specific surgical plan is more complex in clinical experience.

\section{Limitations}

The postoperative residual annular fibrous was not constructed in the stand-alone OLIF model. The muscles were not considered in this study, which play an important role in supporting the stability of lumbar spine. The FEA does not provide actual clinical outcome evidence. Further clinical studies should be done in the future to verify the FEA results.

\section{Conclusions}

The FEA indicated that the OLIF with ASF procedure could not reduce the complication of cage subsidence in the patients with poor bone quality and OLIF with BPSF was very important for the patients with poor bone quality. Since the FEA does not provide actual clinical outcome evidence, further clinical studies should be done in the future to verify the FEA results.

\section{Declarations}

\section{Ethics approval and consent to participate}

This article did not involve the experiments of human and all the data came from the demo file in Mimics 20.0 software.

Consent for publication Not applicable.

\section{Availability of data and material}

The data came from the demo file in Mimics 20.0 software.

\section{Competing interests}

None. 


\section{Funding}

This study was supported by Sanming Project of Medicine in Shenzhen SSZSM201612019■, Shenzhen key Laboratory of digital surgical printing project(ZDSYS201707311542415) and Southern Medical University clinical start-up fund囚LC2016ZD036》.

\section{Authors' contributions}

Dr. GF Fang had full access to all the data in the study and takes responsibility for the integrity of the data and the accuracy of the data analysis. Dr. HX Sang and WH Huang designed the study protocol. SG Chen, WD Zhuang drafted the work.

\section{Acknowledgments}

We would like to thank Mr. Zhang for computer technique support (Guangzhou Li Suen Computer Technology Co., Ltd).

\section{References}

1. Silvestre C, Mac-Thiong JM, Hilmi R, Roussouly P. Complications and morbidities of mini-open anterior retroperitoneal lumbar interbody fusion: oblique lumbar interbody fusion in 179 patients. Asian Spine J. 2012;6:89-97.

2. Quillo-Olvera J, Lin GX, Jo HJ, Kim JS. Complications on minimally invasive oblique lumbar interbody fusion at L2-L5 levels: a review of the literature and surgical strategies. Ann Transl Med. 2018;6(6):101.

3. Li JX, Phan K, Mobbs R. Oblique lumbar interbody fusion: technical aspects, operative outcomes, and complications. World Neurosurg. 2017;98:113-23.

4. Woods KR, Billys JB, Hynes RA. Technical description of oblique lateral interbody fusion at L1-L5 (OLIF25) and at L5-S1 (OLIF51) and evaluation of complication and fusion rates. Spine J. 2017;17:545-53.

5. Fujibayashi S, Kawakami N, Asazuma T, Ito M, Mizutani J, Nagashima H, Nakamura M, Sairyo K, Takemasa R, Iwasaki M. Complications associated with lateral interbody fusion: nationwide survey of 2998 cases during the first two years of its use in Japan. Spine (Phila Pa 1976). 2017;42:147884.

6. Abe K, Orita S, Mannoji C, Motegi H, Aramomi M, Ishikawa T, Kotani T, Akazawa T, Morinaga T, Fujiyoshi T, Hasue F, Yamagata M, Hashimoto M, Yamauchi T, Eguchi Y, Suzuki M, Hanaoka E, Inage K, Sato J, Fujimoto K, Shiga Y, Kanamoto H, Yamauchi K, Nakamura J, Suzuki T, Hynes RA, Aoki Y, Takahashi K, Ohtori S. Perioperative Complications in 155 Patients Who Underwent Oblique Lateral Interbody Fusion Surgery: Perspectives and Indications From a Retrospective, Multicenter Survey.Spine (Phila Pa 1976). 2017;42(1):55-62. 
7. Malham GM, Parker RM, Blecher CM, Seex KA. Assessment and classifification of subsidence after lateral interbody fusion using serial computed tomography. J Neurosurg Spine. 2015;23:589-97.

8. Zeng ZY, Xu ZW, He DW, Zhao X, Ma WH, Ni WF, Song YX, Zhang JQ, Yu W, Fang XQ, Zhou ZJ, Xu NJ, Huang WJ, Hu ZC, Wu AL, Ji JF, Han JF, Fan SW, Zhao FD, Jin H, Pei F, Fan SY, Sui DX. Complications and Prevention Strategies of Oblique Lateral Interbody Fusion Technique. Orthop Surg. 2018;10(2):98-106.

9. Liu J, Feng H. Oblique Lateral Interbody Fusion (OLIF) with Supplemental Anterolateral Screw and Rod Instrumentation: A Preliminary Clinical Study. World Neurosurg. 2020;134:e944-50.

10. Zander T, Dreischarf M, Timm AK, Baumann WW, Schmidt H. Impact of material and morphological parameters on the mechanical response of the lumbar spine - A finite element sensitivity study. $J$ Biomech. 2017;53:185190.

11. Fang G, Lin Y, Wu J, et al. Biomechanical Comparison of Stand-Alone and Bilateral Pedicle Screw Fixation for Oblique Lumbar Interbody Fusion Surgery-A Finite Element Analysis. World Neurosurg. 2020;141:e204-12.

12. Phan $\mathrm{K}$, Maharaj $\mathrm{M}$, Assem $\mathrm{Y}$, et al. Review of early clinical results and complications associated with oblique lumbar interbody fusion (OLIF). J Clin Neurosci. 2016;31:23-9.

13. Mehren C, Mayer HM, Zandanell C, et al. The oblique anterolateral approach to the lumbar spine provides access to the lumbar spine with few early complications. Clin Orthop Relat Res. 2016;474:2020-7.

14. Xie T, Wang C, Yang Z, et al. Minimally Invasive Oblique Lateral Lumbar Interbody Fusion Combined with Anterolateral Screw Fixation for Lumbar Degenerative Disc Disease. World Neurosurg. 2020;135:e671-8.

15. Spiker WR, Goz V. Darrel S. Brodke.Lumbar Interbody Fusions for Degenerative spondylolisthesis: Review of Techniques, Indications, and Outcomes. Global Spine J. 2019;9(1):77-84.

\section{Figures}




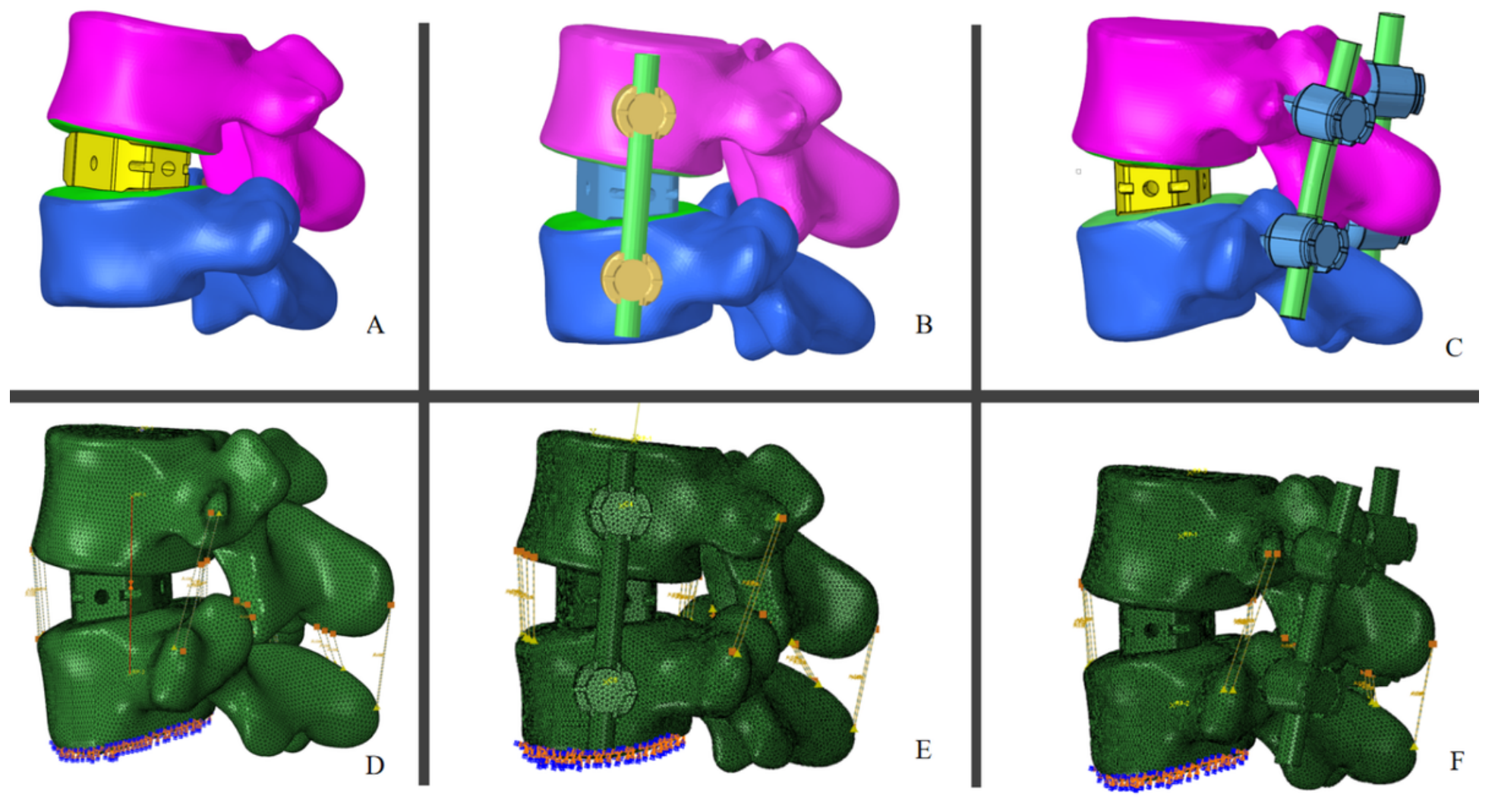

Figure 1

The models of OLIF with SA, BPSF (A:SA geometric model B:ASF geometric model C: BPSF geometric model D: SA FE model E:ASF FE model F: BPSF FE model)

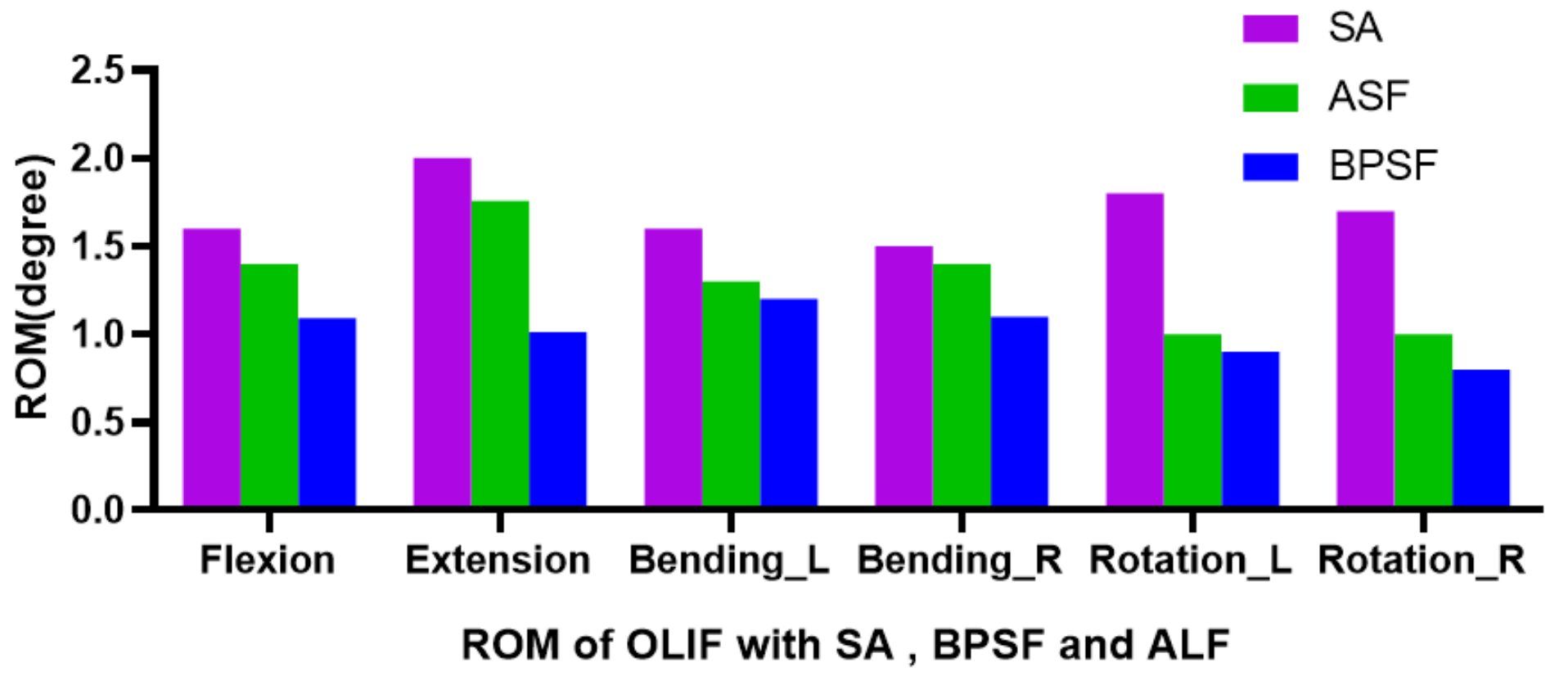

Figure 2

ROM of OLIF with SA, ASF and BPSF 


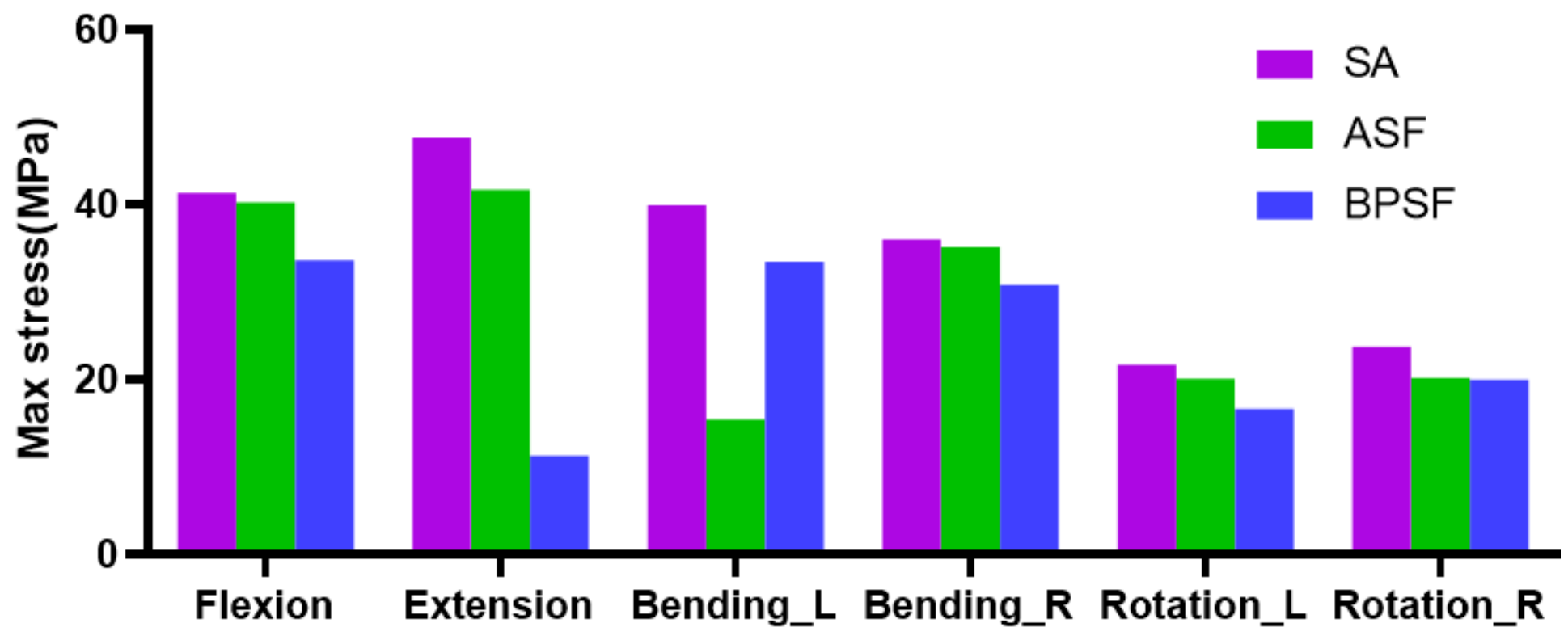

Figure 3

The maximum von Mises stress of L4 IEP in all models

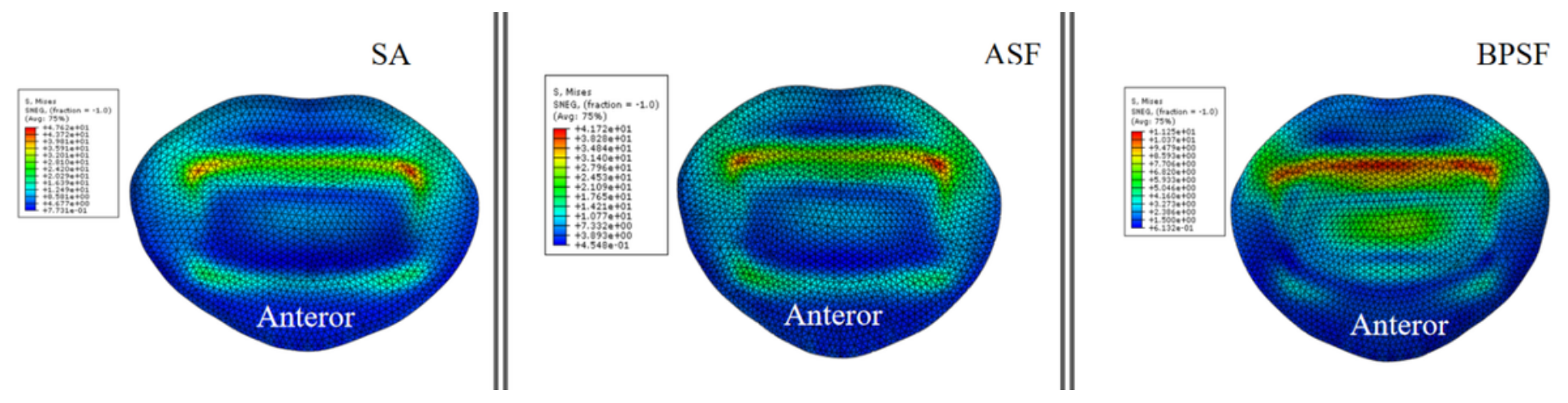

Figure 4

Distribution of maximum stresses and strain in L4 IEP in extension motions 


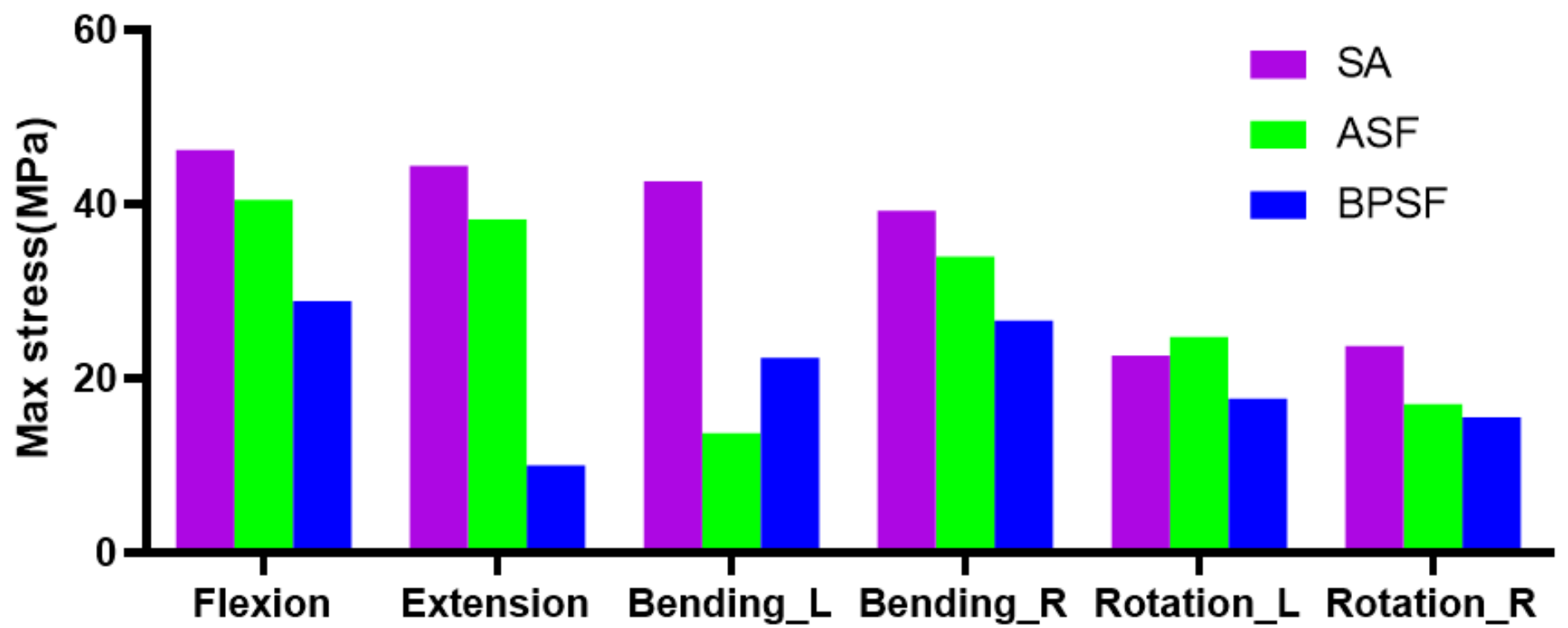

Figure 5

The maximum von Mises stress of L5 SEP in all models

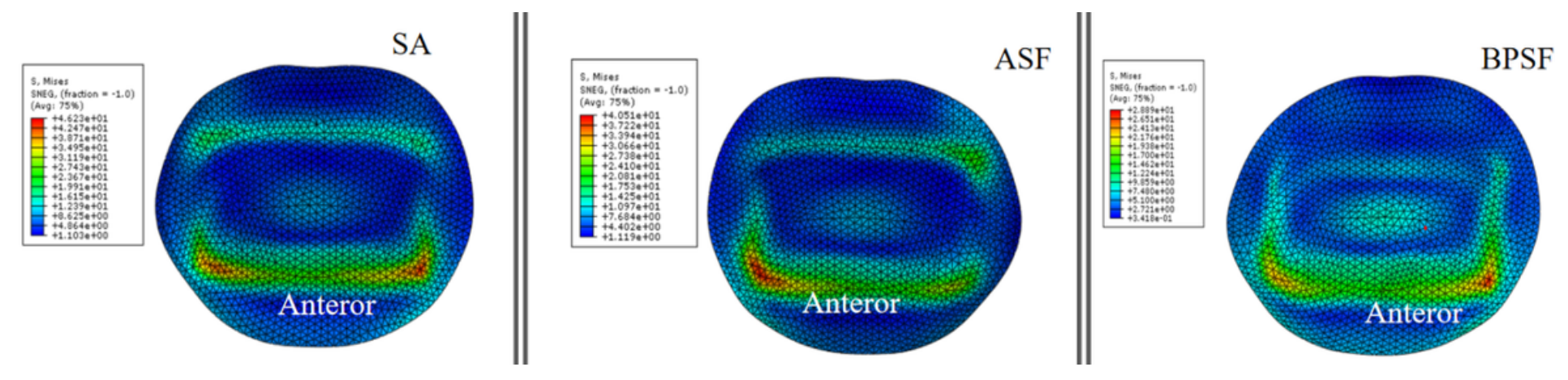

Figure 6

Distribution of maximum stresses and strain in L5 SEP in flexion

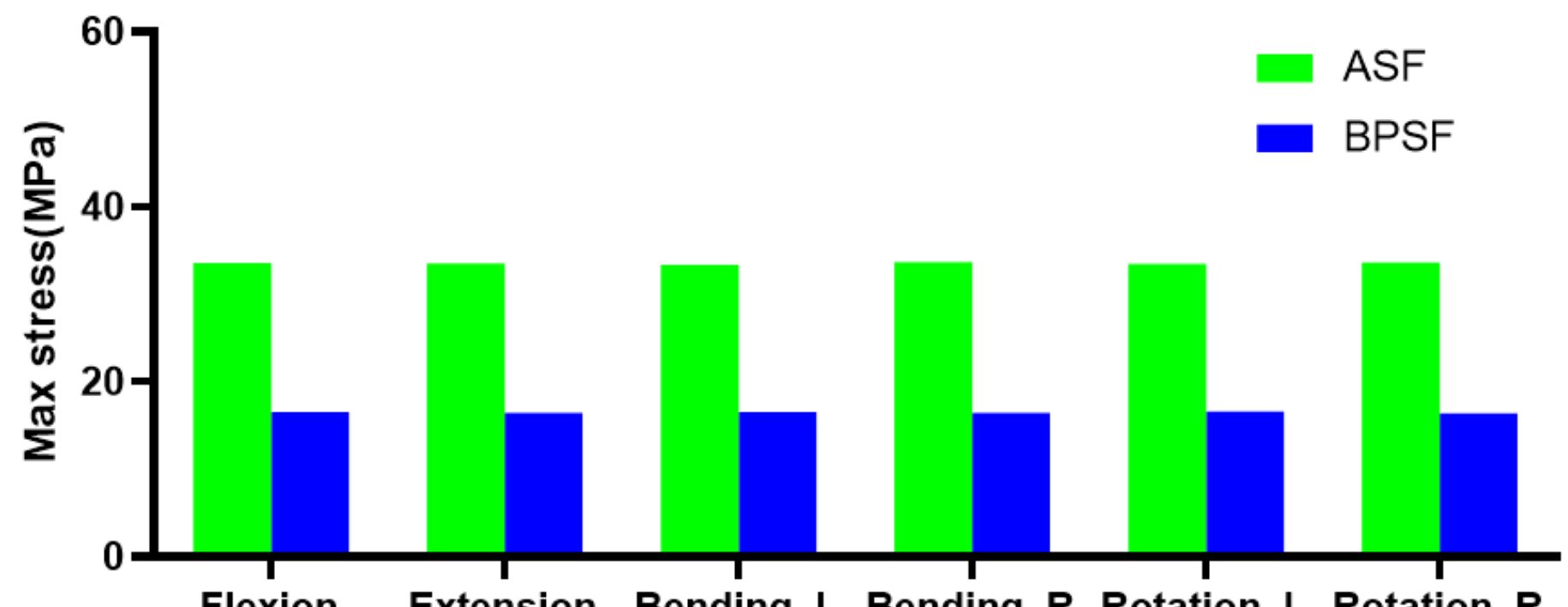

Flexion Extension Bending_L Bending_R Rotation_L Rotation_R 
Figure 7

The maximum von Mises stress of CSVS in all models

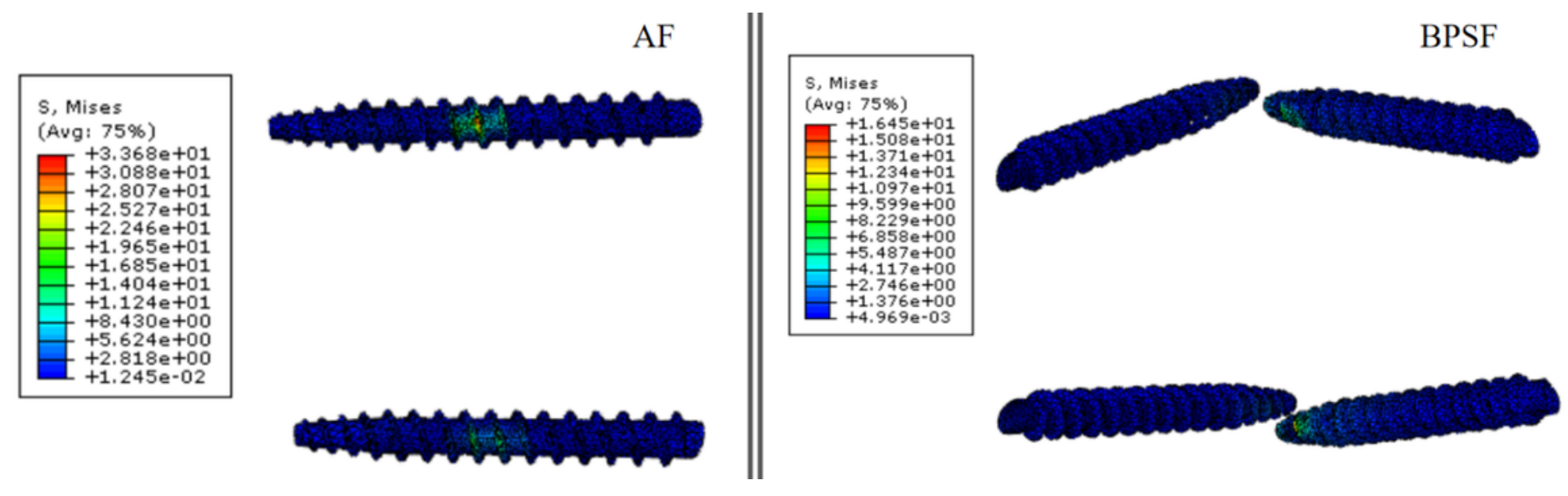

Figure 8

Distribution of maximum stresses of CSVS in flexion moment

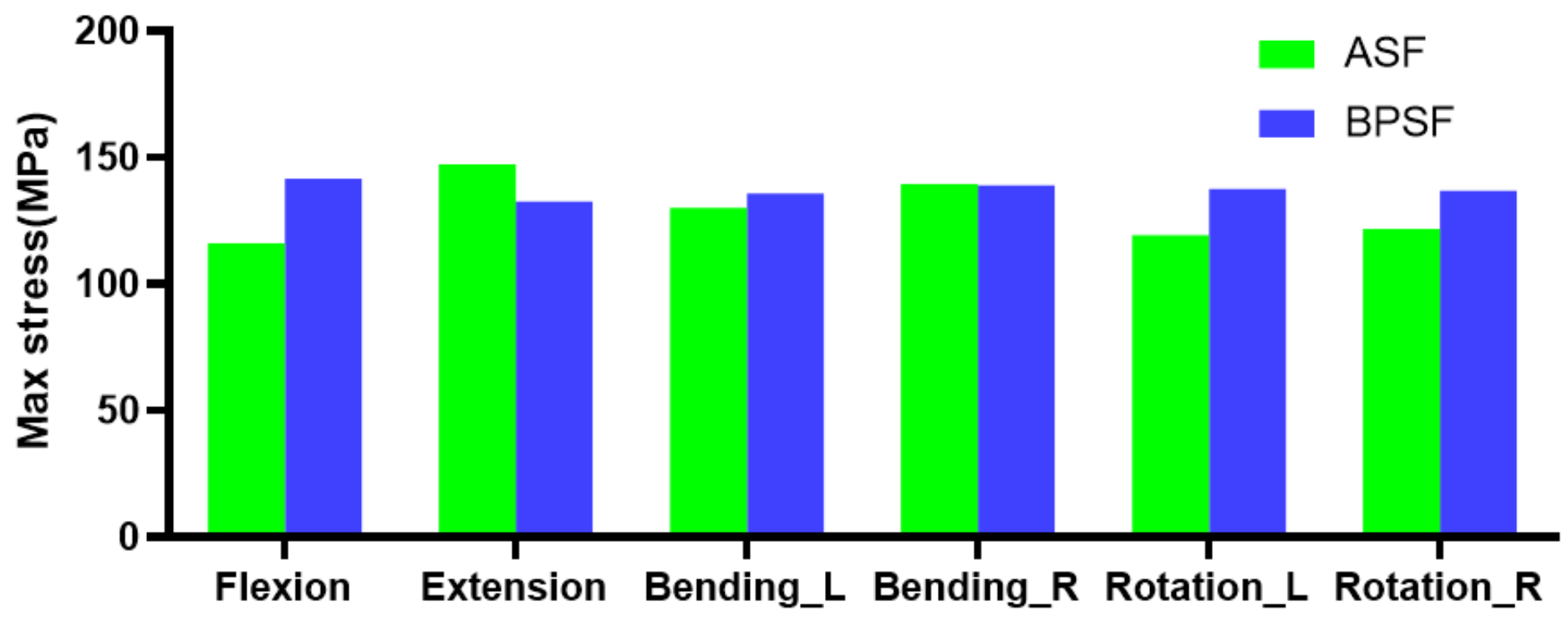

Figure 9

The maximum von Mises stress of screw in all models 

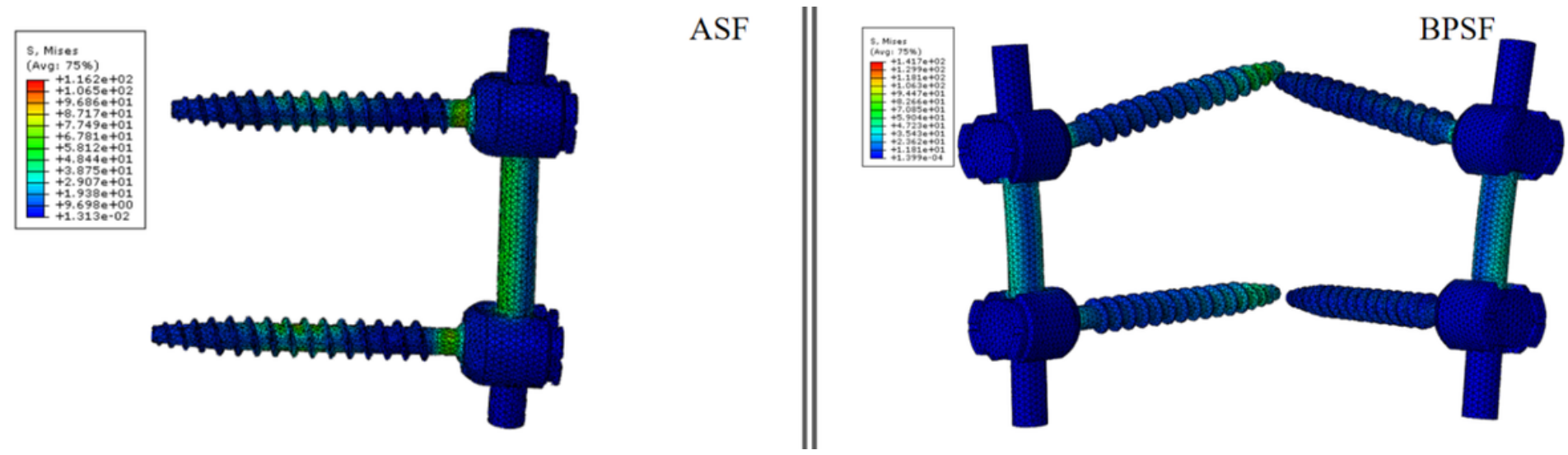

Figure 10

Distribution of maximum stresses of screw in flexion moment

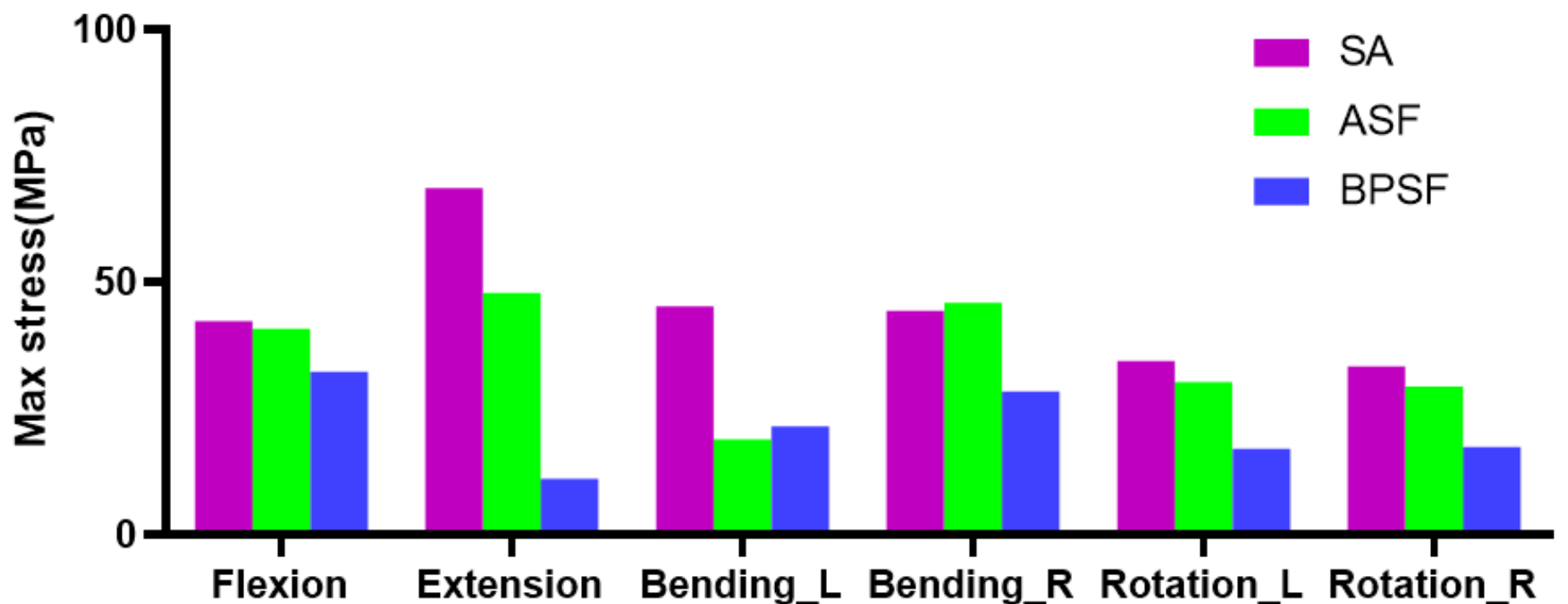

Figure 11

The maximum von Mises stress of cage in all models

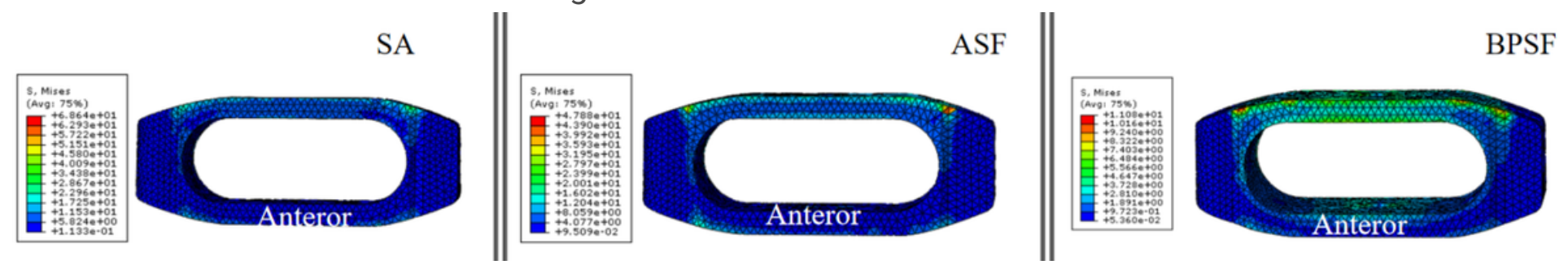

Figure 12

Distribution of maximum stresses of cage in extension moment 

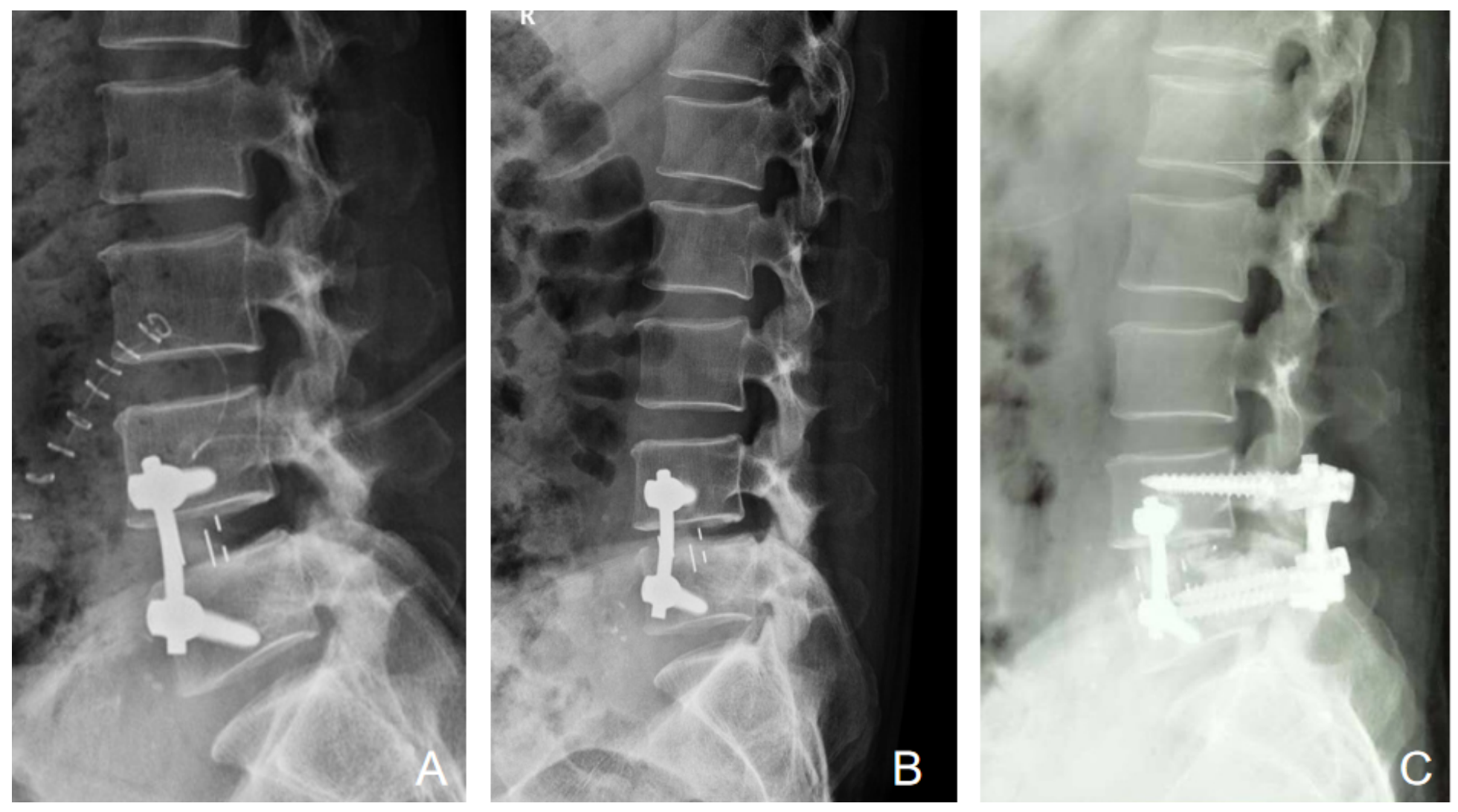

Figure 13

A 65 year-old patient who underwent OLIF with ASF suffered from cage subsidence one month after surgery and revised with BPSF 2 months later.(A: lateral X-ray image of lumbar 2 days after surgery B:lateral X-ray image of lumbar 1 month after surgery $\mathrm{C}$ :lateral X-ray image of lumbar 2 month after surgery) 